\title{
The Role of Cellulose and O-Antigen Capsule in the Colonization of Plants by Salmonella enterica
}

\author{
Jeri D. Barak, ${ }^{1}$ Courtney E. Jahn, ${ }^{2}$ Deanna L. Gibson, ${ }^{3,4}$ and Amy O. Charkowski ${ }^{2}$ \\ ${ }^{1}$ Produce Safety and Microbiology Research Unit, United States Department of Agriculture-Agricultural Research Service, \\ Albany, CA 94710, U.S.A.; ' ${ }^{2}$ epartment of Plant Pathology, Rm 682 Russell Laboratories, 1630 Linden Dr., University \\ of Wisconsin-Madison, Madison, WI 53706, U.S.A.; ${ }^{3}$ Department of Biochemistry and Microbiology, University of Victoria, \\ Victoria V8W 3P6, British Columbia, Canada; ${ }^{4}$ Department of Pediatrics, University of British Columbia, Vancouver V5Z 4H4, \\ British Columbia, Canada
}

Submitted 16 November 2006. Accepted 17 April 2007.

\begin{abstract}
Numerous salmonellosis outbreaks have been associated with vegetables, in particular sprouted seed. Thin aggregative fimbriae (Tafi), a component of the extracellular matrix responsible for multicellular behavior, are important for Salmonella enterica attachment and colonization of plants. Here, we demonstrate that the other surface polymers composing the extracellular matrix, cellulose, and $\mathrm{O}$-antigen capsule also play a role in colonization of plants. Mutations in bacterial cellulose synthesis (bcsA) and $O$-antigen capsule assembly and translocation (yihO) reduced the ability to attach to and colonize alfalfa sprouts. A colanic acid mutant was unaffected in plant attachment or colonization. Tafi, cellulose synthesis, and $\mathrm{O}$-antigen capsule, all of which contribute to attachment and colonization of plants, are regulated by AgfD, suggesting that AgfD is a key regulator for survival outside of hosts of Salmonella spp. The cellulose biosynthesis regulator adrA mutant was not affected in the ability to attach to or colonize plants; however, promoter probe assays revealed expression by cells attached to alfalfa sprouts. Furthermore, quantitative reverse-transcriptase polymerase chain reaction revealed differential expression of $a g f D$ and $a d r A$ between planktonic and plant-attached cells. In addition, there was no correlation among mutants between biofilm formation in culture and attachment to plants. Outside of animal hosts, $S$. enterica appears to rely on an arsenal of adhesins to persist on plants, which can act as vectors and perpetuate public health concerns.
\end{abstract}

Additional keywords: curli, GGDEF, lipopolysaccharide.

The gram-negative bacterium Salmonella enterica causes salmonellosis, a foodborne illness that results in roughly 50,000 cases and 50 fatalities per year in the United States (Hopkins et al. 2005). Numerous S. enterica outbreaks have been associated with contaminated sprouts and melon and tomato fruit, and the incidence of reported contamination has remained constant despite industry attempts to eliminate sources of contamination and sanitize seed prior to sprouting (Beuchat 2002).

Corresponding author J. D. Barak: E-mail: jbarak@pw.usda.gov; Telephone: +1.510 .559 .6180 ; Fax: +1.510 .559 .6162 .

This article is in the public domain and not copyrightable. It may be freely reprinted with customary crediting of the source. The American Phytopathological Society, 2007.
S. enterica has been found colonizing seed (Mahon et al. 1997; Winthrop et al. 2003), sprouts (O’Mahony et al. 1990), leaves (Campbell et al. 2001), and fruit (Guo et al. 2001; Mohle-Boetani et al. 1999) of a variety of plant species. However, few studies have addressed the molecular mechanisms for attachment and colonization of plants by human bacterial pathogens. Recently, we found that genes required for animal virulence of $S$. enterica also are required for attachment and colonization of sprouts. These include the alternative sigma factor $r p o S$ and $a g f D$, which is important for the synthesis of thin aggregative fimbriae (Tafi; also known as curli in Escherichia coli) (Barak et al. 2005). RpoS transcribes agfD, which then positively regulates the agf (Tafi) operon (Romling et al. 2000). Also identified to be important for attachment to alfalfa sprouts in our previous study was $a g f B$, the cell-bound fimbrial subunit that anchors Tafi to the cell surface (Hammar et al. 1996) and nucleates polymerized fibers composed of both AgfA and AgfB (White et al. 2001). Production of curli, a Tafi homolog in E. coli, also has been identified as important for attachment of $E$. coli to alfalfa sprouts (Jeter and Matthysse 2005).

In addition to its positive regulation of Tafi, AgfD also has been reported to regulate cellulose (Romling et al. 1998; Zogaj et al. 2001) and O-antigen (O-Ag) capsule (Gibson et al. 2006) production in $S$. enterica. All three of these components constitute the extracellular matrix. In addition, Tafi and cellulose are required for aggregative multicellular behavior (Romling et al. 2003). The discovery of a function for Tafi in plant colonization for $S$. enterica led us to question whether other components of the extracellular matrix in S. enterica (i.e., cellulose and $\mathrm{O}-\mathrm{Ag}$ capsule) are involved in plant attachment and colonization. In animal pathogens, cellulose has been shown to participate in biofilm formation, multicellular behavior, adherence to animal cells, or stress tolerance (Ledeboer et al. 2006; Scher et al. 2005; White et al. 2003, 2006). The O-Ag capsule has been found to be important for desiccation tolerance (Gibson et al. 2006). Colanic acid is another surface polymer that has been found associated with curli in E. coli (Prigent-Combaret et al. 2000). Colanic acid and Tafi both play a role in $S$. enterica biofilms on animal cells (Ledeboer and Jones 2005).

In this study, we focused on genes necessary for the production of components of the extracellular matrix to ask whether these surface features play a role in alfalfa sprout attachment or colonization. These genes also have been described previously as components of multicellular behavior, involved in cell-cell interactions, or required for persistence outside a host. These genes are $b c s A$, which encodes a cellulose synthase, and 
$a d r A$, which mediates production of the allosteric activator cdi-GMP and regulates cellulose biosynthesis under some conditions. Also included in the study was a $b c s A$ /agf $B$ double mutant. The $b \operatorname{cs} A$ mutant does not produce cellulose and the bcsA/agfB mutant produces neither cellulose nor Tafi. The contribution of colonic acid and O-Ag capsule to plant attachment was studied with strains mutated in wcaJ, which encodes an undecaprenylphosphate glucose phosphotransferase required for colanic acid production, and yihO, which encodes a glucuronide transporter required for capsule transport to the cell surface. Each of these mutants lacks production of their respective surface polymer. To examine initial attachment to plants, 3-day-old alfalfa sprouts were inoculated and attached cells were enumerated $4 \mathrm{~h}$ later. To examine colonization of plants, alfalfa seed were inoculated and attached bacterial populations were enumerated at 24 and $48 \mathrm{~h}$ postinoculation (hpi). To discover the role of $a d r A$ in regulation of cellulose production by $S$. enterica on plants, quantitative reverse-transcriptase polymerase chain reaction (qRT-PCR) was used to quantify the expression of $b c s A$ in the wild type and an $a d r A$ mutant. In addition, expression of $a g f D$, which encodes an extracellular matrix regulator, $b c s A$, and $a d r A$ were examined in planta with a promoter probe assay.

\section{RESULTS}

\section{S. enterica cellulose biosynthesis contributes}

to bacterial attachment to and colonization of plants.

The ability of the $S$. enterica wild type and mutants to exhibit multicellular behavior as manifested by the formation of pellicles on the culture surface and biofilms at the air-liquid interface of glass test tubes was determined in Luria-Bertani broth, no salt (LBNS) (Fig. 1) and compared with their ability to colonize plants (Figs. 2 and 3). As previously reported (Romling et al. 2000; Solano et al. 2002), the mutants associ- ated with cellulose biosynthesis, including $a d r A$ (digunylatecyclase), bcsA (cellulose synthase) and $b c s A / a g f B$ (cellulose synthase and fimbriae nucleator), could not form wild-type biofilms at the air-liquid interface (Fig. 1).

Although it could not form a pellicle at the air-liquid interface, the $a d r A$ mutant colonized alfalfa sprouts as well as the wild type at 24 and 48 hpi (Fig. 2A). Fewer cells of the $b \operatorname{cs} A$ mutant colonized alfalfa sprouts than the wild type at both 24 and $48 \mathrm{hpi}$ and even fewer cells of the $b c s A / a g f B$ double mutant were recovered from alfalfa sprouts at 48 hpi (Fig. 2B) $(P<$ $0.05)$. When the bcsA mutation in $S$. enterica Enteritidis $27655-$ $3 \mathrm{~b}$ was complemented on a plasmid (pJDB2 containing $b c s A$ ), the ability to colonize 24 and 48 hpi was restored to wild-type levels (Fig. 2B). An $a g f B$ mutant previously had been shown to be impaired in attachment to sprouts (Barak et al. 2005); thus, both Tafi and cellulose, but not the cellulose regulator $a d r A$, play roles in $S$. enterica colonization of sprouts.

To discriminate between the roles of the multicellular matrix in initial attachment versus colonization, 3-day-old sprouts were inoculated with the wild type or mutants and the sprouts were sampled $4 \mathrm{~h}$ later, modeling bacterial initial attachment. Fewer cells of the $b c s A / a g f B$ double mutant were attached to sprouts than the $b c s A$ mutant and both mutants were reduced compared with the wild type (Fig. $2 \mathrm{C})(P<0.05)$. When the $b c s A$ mutation was complemented by expression of $b c s A$ from a plasmid, bacterial attachment was only partially restored to wild-type level; however, it was significantly higher than the bcs $A$ mutant $(P<0.01)$ (Fig. $2 \mathrm{C})$.

\section{Lipopolysaccharide O-Ag capsule is important for bacterial attachment to and colonization of plants.}

The $S$. enterica Enteritidis strain used in this study produces lipopolysaccharide $\mathrm{O}-\mathrm{Ag}$ capsule and colanic acid. To uncover the role of $\mathrm{O}-\mathrm{Ag}$ capsule and colanic acid in plant colonization, alfalfa seed were inoculated with the wild type, yihO

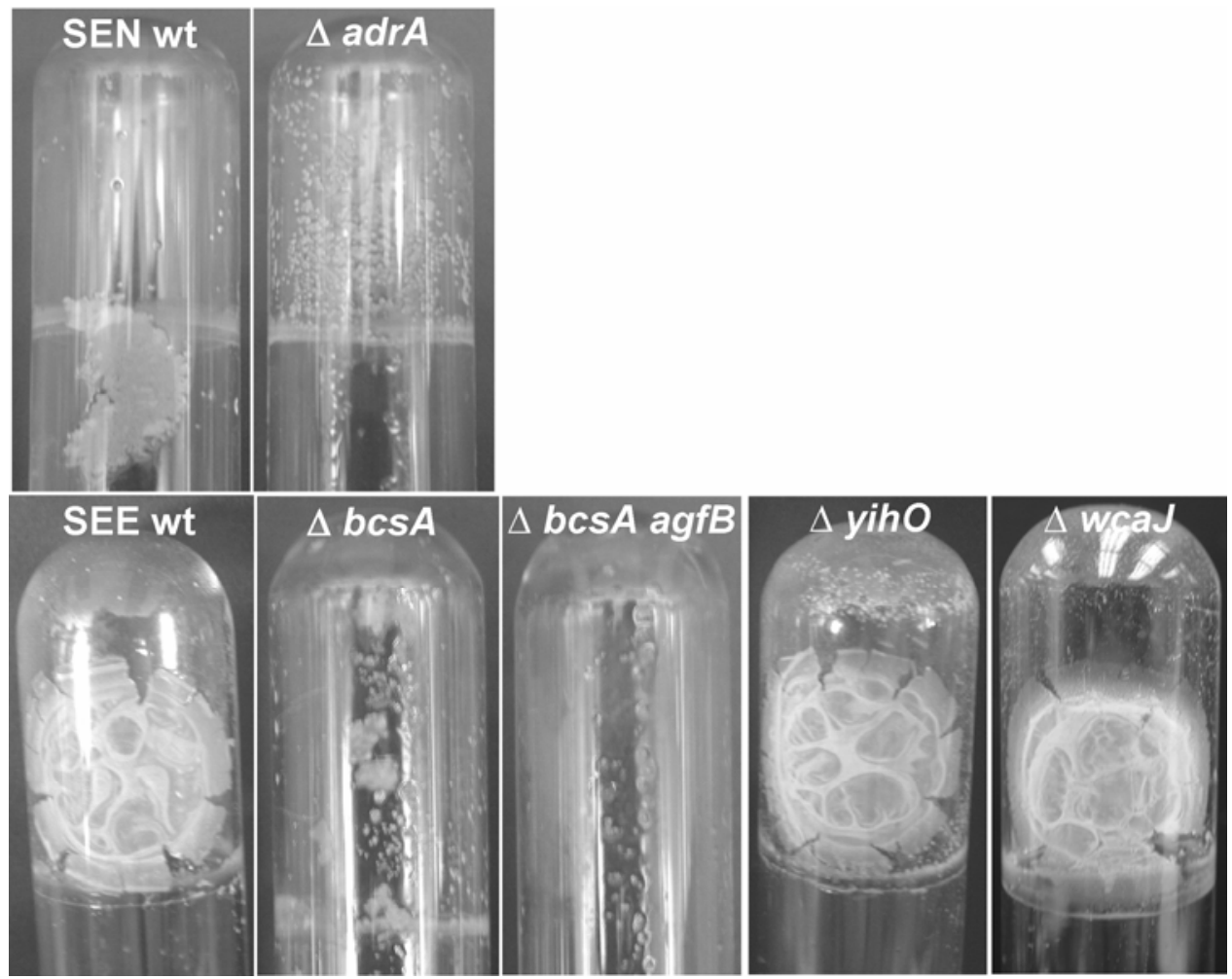

Fig. 1. Photographs of 72-h-old biofilms and pellicles grown at the air-liquid interface of Luria-Bertani broth, no salt cultures in test tubes. The medium was removed and the biofilms and pellicles were retained in the test tubes. Upper row: the Salmonella enterica Newport wild type and adrA mutant; lower row: $S$. enterica Enteritidis wild type, bcsA mutant, bcsA agfB mutant, yihO mutant, and wcaJ mutant. 
(capsule assembly and transport), or wcaJ (a transferase in the colanic acid gene cluster) mutants, and alfalfa sprouts were sampled. Colonization at both 24 and 48 hpi was reduced for the yihO mutant compared with the wild type (Fig. 3A) $(P<$ $0.05)$, whereas the wcaJ mutant colonized sprouts as well as the wild type. To discriminate between the roles of surface polymers in initial attachment versus colonization, 3-day-old sprouts were inoculated with wild-type or mutant cells and sprouts were sampled $4 \mathrm{~h}$ later. The yihO mutant attached at lower levels than the wild type (Fig. 3B) $(P<0.05)$ and there was no difference between the wild type and wcaJ mutant (data not shown)

\section{S. enterica cellulose synthesis genes are expressed} during colonization of sprouts and $a d r A$ is

differentially regulated in planktonic and attached cells.

To visualize gene expression in vivo, the promoters of the regulators $a g f D, a d r A$, and the enzyme $b c s A$ were cloned upstream of the green fluorescent protein (GFP) gene in pProbe$\mathrm{GT}^{\prime}$ and the plasmids were transformed into $S$. enterica Newport cells. Alfalfa seed were inoculated with the $S$. enterica Newport strains and sprouts were examined 48 hpi. The sprouts were visualized with a DNA stain and the $S$. enterica cells were visualized with an antibody bound to a fluorescent label. S. enterica Newport cells predominately colonized the
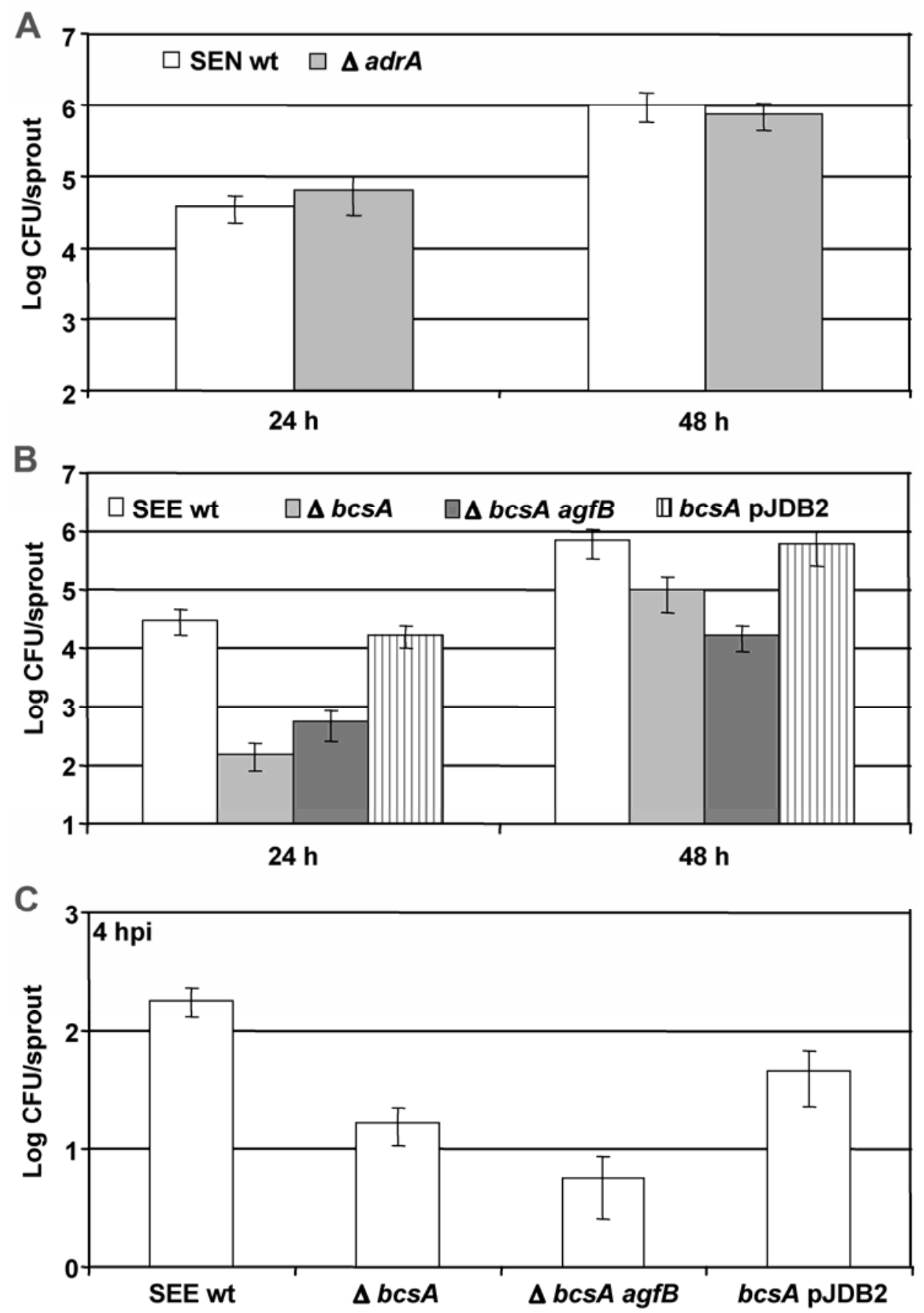

Fig. 2. Salmonella enterica wild-type and cellulose biosynthesis mutant colonization and attachment assays. A, S. enterica Newport wild-type (SEN wt) and $a d r A$ mutant CFU recovered from alfalfa sprouts 24 and $48 \mathrm{~h}$ post-seed inoculation. B, S. enterica Enteritidis wild type (SEE wt), bcsA and bcsA/agfB mutants, and complimented $b c s A$ mutant $\triangle b c s A$ (pJDB2) CFU recovered from alfalfa sprouts 24 and $48 \mathrm{~h}$ post-seed inoculation. C, SEE wt, $b c s A$ and $b c s A / a g f B$ mutants, and complimented bcsA mutant CFU recovered from mature alfalfa sprouts $4 \mathrm{~h}$ postinoculation. The data shown are from one of three representative experiments, with 10 sprouts sampled from each treatment. Standard deviation is represented as \pm error bars. 
root hairs of the alfalfa sprouts, as previously reported (Charkowski et al. 2002). The reporter plasmids did not appear to inhibit bacterial colonization because similar numbers of $S$. enterica Newport cells colonized individual alfalfa sprouts regardless of the plasmid carried (data not shown). When the sprouts were examined with confocal laser scanning microscopy, green fluorescent cells were observed in the strains carrying each of the promoter reporters, agfD, adrA, and $b c s A$, demonstrating that all three of these genes are expressed in cells attached to alfalfa roots (Fig. 4). The highest number of green fluorescent cells was observed in the strain carrying the $a g f D$ promoter reporter, followed by $a d r A$ and $b c s A$ strains. Therefore, a higher percentage of cells appear to be expressing the two regulatory genes than the cellulose biosynthesis operon.

The GFP promoter probes indicated that both $a d r A$ and $b c s A$ genes were expressed on plants, yet the cellulose synthase bcs $A$ and the cellulose regulator $a d r A$ mutants had different attachment and colonization phenotypes on alfalfa sprouts. For a more quantitative assessment of expression and regulation, we examined $b \operatorname{cs} A$ and $a d r A$ gene expression during alfalfa sprout colonization to determine whether $a d r A$ affected $b c s A$ expression. qRT-PCR revealed that $a d r A$ expression in planktonic cells is 64-fold higher than in cells attached to alfalfa sprouts (Table 1). The $b c s A$ gene also was differentially expressed, but to a lesser extent, with only a twofold difference observed between planktonic and attached cells. Thus, large changes in $a d r A$ expression do not result in correspondingly large changes in $b c s A$ expression. In both cases, the single-copy 50S ribosomal subunit protein $\mathrm{L} 21(\mathrm{rplU})$ was used as a reference gene for expression studies because its PCR primer set had a similar efficiency of $70 \%$ to the $b c s A$ and $a d r A$ primer sets and it has consistent expression under these conditions (data not shown).

To further elucidate the role of $a d r A$ in cellulose biosynthesis, qRT-PCR was used to monitor the expression of $b c s A$ in the wild type and $a d r A$ mutant. The strains were grown in conditions known to promote cellulose production (Garcia et al. 2004): static cultures of LBNS at $28^{\circ} \mathrm{C}$. ompA, outer membrane protein $\mathrm{A}$, was used as a reference gene for expression studies because its PCR primer set had a similar efficiency of $70 \%$ to the $b c s A$ primer set and it has consistent expression under these conditions (data not shown). First, RNA was extracted from static cultures of $S$. enterica Newport grown for 5, 10, 12, and $17 \mathrm{~h}$ to determine the time of peak $b c s A$ expression (data not shown). $b c s A$ expression was highest in 12-h-old cultures. RNA then was extracted from both the wild type and $a d r A \mathrm{mu}-$ tant once per hour from 12 to $16 \mathrm{~h}$, and the ratio of $b c s A$ expression in the wild type versus the $a d r A$ mutant ranged from 0.56 to 0.83 compared with the housekeeping gene ompA (Table 2). The ratio of bcsA expression between the wild type and $a d r A$ mutant cells was $<1$; therefore, these results suggest that $b c s A$ expression was slightly higher in the $a d r A$ mutant than the wild type. In addition, because the change in $b c s A$ expression was relatively small, these data do not support a role
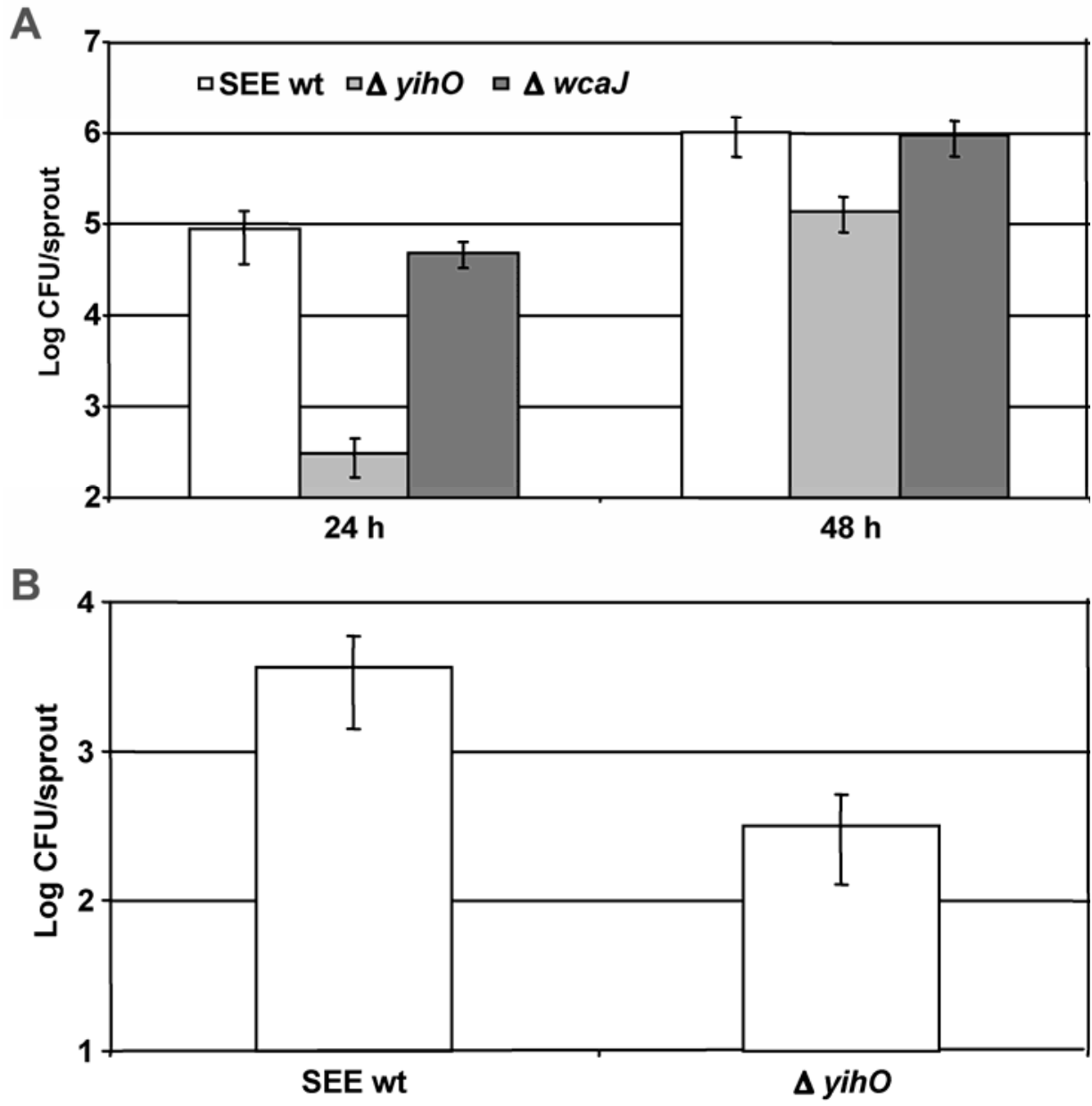

Fig. 3. Salmonella enterica wild-type and extracellular matrix biosynthesis mutant colonization and attachment assays. A, S. enterica Enteritidis wild-type (SEE wt) and $y i h O$ and wcaJ mutant CFU recovered from alfalfa sprouts 24 and $48 \mathrm{~h}$ post-seed inoculation. B, SEE wt and $y i h O$ mutant $\mathrm{CFU}$ recovered from 3-day-old alfalfa sprouts following 4-h attachment assays. The data are from one of three representative experiments with 10 sprout samples taken from each treatment. Standard deviation is represented as \pm error bars in all panels. 
for AdrA as a transcriptional regulator of the bcs operon, at least under these conditions.

\section{DISCUSSION}

In a previous study regarding the genes necessary for $S$. enterica interactions with alfalfa sprouts, Tafi was identified as a contributor to plant colonization by the identification of $a g f D$ (extracellular matrix regulator) and $a g f B$ (Tafi nucleator) from a random screen of mutants (Barak et al. 2005). The contribution of Tafi to $S$. enterica plant colonization pointed to the question of whether other components of the extracellular matrix or multicellular behavior also are involved in S. enterica colonization of plants. The results of the present study reveal a role for cellulose and O-Ag capsule, but not colanic, in attachment to and colonization of plants under the conditions studied here.

We first focused on bacterial cellulose synthesis because cellulose and Tafi genes often are co-expressed and share the regulator AgfD (Romling 2005). We chose to use a mutant of the bacterial cellulose synthase gene, $b c s A$, because it is one of the few $b c s$ genes with a known function. The $b c s A$ mutant was reduced in both initial attachment to alfalfa sprouts and colonization over time. We also found that a $b \operatorname{cs} A / a g f B$ double mutant was further reduced compared with the $b \operatorname{cs} A$ mutant, suggesting that the contributions of cellulose and Tafi in colonization of plants are additive. Therefore, cellulose and Tafi, which are required for multicellular behavior (Romling et al. 1998), also play a role in survival outside of animal hosts via colonization of plants.

The plant-pathogenic bacterium Agrobacterium tumefaciens (Matthysse and McMahan 1998) and plant-associated bacterium Rhizobium leguminosarum bv. trifolii (Ausmees et al. 1999) use cellulose to adhere to plant tissue, specifically root tissue. We have observed previously (Charkowski et al. 2002) and in this study that $S$. enterica preferentially colonizes roots and uses cellulose to adhere. Therefore, we hypothesize that human pathogens and plant-associated bacteria use at least one of the same extracellular molecules, cellulose, to colonize plants, and its role in colonization probably is involved in cellto-cell interactions within a biofilm.

Surface polymers play essential roles in the survival of bacterial pathogens outside of hosts. For example, Tafi and cellulose have been shown to confer resistance to bleach (Scher et al. 2005, White et al. 2006), whereas the O-Ag capsule has been shown to be important in desiccation tolerance, thus promoting environmental persistence (Gibson 2006).

Table 1. Average expression of Salmonella enterica Newport genes relative to $r p l U$ in planktonic and attached cells

\begin{tabular}{lcc}
\hline & \multicolumn{2}{c}{ Condition } \\
\cline { 2 - 3 } Gene & Planktonic & Attached $^{\mathbf{a}}$ \\
\hline$a d r A$ & $4,096 \pm 0$ & $64 \pm 0$ \\
$b c s A$ & $266 \pm 91$ & $107 \pm 37$ \\
\hline
\end{tabular}

${ }^{a}$ Cells attached to alfalfa sprouts.

Table 2. Ratio of bcsA expression in the Salmonella enterica Newport wild type versus the adrA mutant, relative to ompA expression, in cells grown in static liquid Luria-Bertani broth, no salt

\begin{tabular}{lcc}
\hline Time (hour) & Average & Standard deviation \\
\hline 12 & 0.42 & 0.28 \\
13 & 0.54 & 0.42 \\
14 & 0.44 & 0.18 \\
15 & 0.66 & 0.15 \\
16 & 0.66 & 0.24 \\
\hline
\end{tabular}
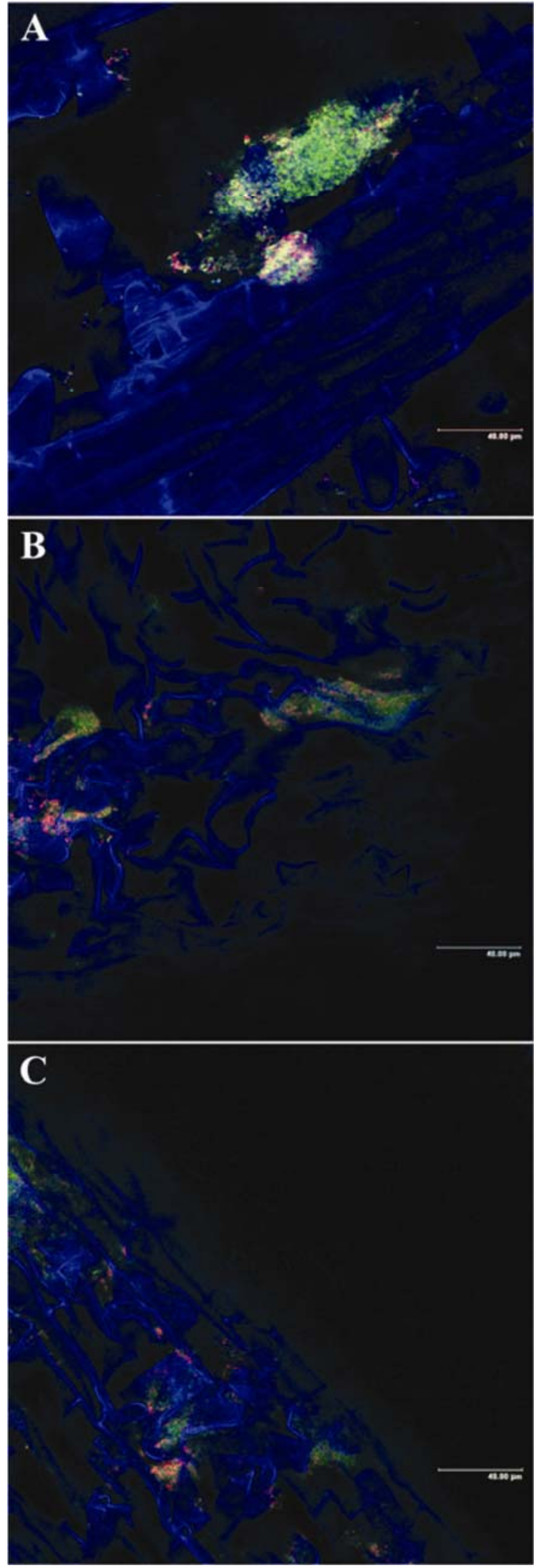

Fig. 4. Micrographs of confocal laser scanning microscopy projected $z$ series of promoter probe assays of Salmonella enterica colonizing alfalfa sprouts. Sprouts were visualized using the nucleic acid stain SYTO59 (blue). S. enterica cells were localized by indirect immunofluorescence with anti-Salmonella CSA-1and an Alexa 568-conjugated rabbit anti-goat immunoglobulin G secondary antibody (red). Cells were intrinsically labeled by the plasmid pProbeGT', which carries the gene encoding the green fluorescent protein transcribed from $\mathbf{A}$, the $a f g D, \mathbf{B}$, the $a d r A$, or $\mathbf{C}$, the $b c s A$ promoter. 
Here, we found that $\mathrm{O}-\mathrm{Ag}$ capsule also aids in persistence by promoting attachment to and colonization of plants, perhaps as vectors between hosts. Furthermore, O-Ag, Tafi, and cellulose are all regulated, directly or indirectly, by AgfD, suggesting that this $S$. enterica regulator is of most importance outside the animal host.

Unexpectedly, mutation of the cellulose regulator $a d r A$ did not affect bacterial adherence or colonization of plants even though the cellulose synthesis gene $b \operatorname{cs} A$ was required for adhesion and colonization. Thus, we investigated whether the expression of bcsA was impaired in the adrA mutant under conditions known to require AdrA. Our biofilm assays showed that $S$. enterica Newport requires AdrA for pellicle formation in LBNS (Fig. 1) similar to S. enterica Typhimurium (Garcia et al. 2004). Thus, $a d r A$ is not required for bacterial cellulose synthesis by $S$. enterica on plants. In $S$. enterica, several other GGDEF-domain-containing proteins can activate cellulose biosynthesis and compensate for an adrA mutation (Garcia et al. 2004; Simm et al. 2004). Our qRT-PCR results and promoter probe assay demonstrated that $a d r A$ and $b c s A$ are expressed when $S$. enterica is attached to alfalfa sprouts. We conclude that $a d r A$ is expressed in planta, but that its role in regulation of cellulose biosynthesis can be accomplished by other proteins in its absence, unlike when grown in LBNS. The plant environment may be more similar to the carbon-source-rich, traceelement-poor medium where $S$. enterica Typhimurium requires the GGDEF protein GcpA, and not AdrA, for cellulose production (Garcia et al. 2004).

Our qRT-PCR results revealed differential expression of $a d r A$ between attached and planktonic cells in contrast to what we observed with $b c s A$ expression, which was only slightly upregulated in planktonic cells. This suggests that, in planta,
AdrA may regulate more than c-di-GMP levels (Simm et al. 2004) for cellulose biosynthesis. In addition to multicellular behavior, c-di-GMP regulation affects interactions between bacteria and their eukaryotic hosts and other phenotypes (Romling et al. 2005). AdrA may play a role in the colonization of plants by $S$. enterica; however, its role may be difficult to elucidate with the ability of $S$. enterica to utilize other GGDEF proteins in the absence of AdrA.

Many studies focus on the altered ability of mutant strains to form biofilms or pellicles on abiotic surfaces as a screen for altered in vivo activity. Our results show that biofilm and pellicle formation in vitro are not correlated with bacterial attachment or colonization of plants. Both the yihO and wcaJ mutants could form a pellicle; however, the yihO mutant was unable to colonize alfalfa sprouts as well as wild-type cells and the wcaJ mutant had no phenotype on plants. Thus, the bacterial requirements for attaching to or colonizing of plant tissue differ significantly from what is required to attach to glass test tubes or other bacterial cells in vitro and attachment to and colonization of animal cells (e.g., colanic acid). In addition, because $a g f D, a g f B$, and $b c s A$ all are downregulated when $S$. enterica cells are attached to plant tissue, it seems likely that genes important for attachment or colonization to surfaces would not be found by gene expression methods that search for genes upregulated in attached cells. In total, Tafi, cellulose, and O-Ag capsule all appear to play a role in S. enterica attachment to and colonization of plants, although no individual polymer is required for either phenotype. Together, these $S$. enterica surface molecules appear to aid this human pathogen in using plants as vectors between hosts and in perpetuating the public health risk of human pathogen contamination of fresh produce.

Table 3. Strains and plasmids used in this study

\begin{tabular}{|c|c|c|}
\hline Strains and plasmids & Description $^{a}$ & Source \\
\hline \multicolumn{3}{|c|}{ Salmonella enterica Newport } \\
\hline 96E01152C-TX & Isolated from alfalfa seed & Inami and Moler 1999 \\
\hline 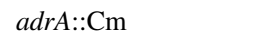 & $\mathrm{Cm}^{\mathrm{r}}$ fragment inserted into NarI site of $a d r A$ & This study \\
\hline \multicolumn{3}{|l|}{ S. enterica Enteritidis } \\
\hline $27655-3 b$ & Clinical isolate & Collinson et al. 1991 \\
\hline$\Delta b c s A$ & In-frame deletion removing nucleotides encoding amino acids $165-828$ in BcsA & White et al. 2003 \\
\hline$\Delta b c s A \Delta a g f B$ & $\begin{array}{l}\text { In-frame deletions removing nucleotides encoding amino acids } 165-828 \text { in BcsA and } \\
48-108 \text { in AgfB }\end{array}$ & William Kay \\
\hline$\Delta y i h O$ & In-frame deletion removing nucleotides encoding amino acids 7-106 in YihO & Gibson et al. 2006 \\
\hline$\Delta w c a J$ & In-frame deletion removing nucleotides encoding amino acids $34-436$ in WcaJ & White et al. 2003 \\
\hline JDB 553 & $\Delta b c s A \mathrm{pJDB} 2$ & This study \\
\hline \multicolumn{3}{|l|}{ Plasmids } \\
\hline pJDB2 & $\begin{array}{l}\text { pBBR } 1:: b c s A ; 2,662-b p \text { fragment containing } b c s A \text { cloned from } 27655-3 b \text { using primers } \\
\text { bcsA-fwd-1145 and bcsA-rev-3797 and inserted into pBBR1 }\end{array}$ & This study \\
\hline pTadrA & $\begin{array}{l}\text { 4.8-kb fragment containing } a d r A \text { amplified with primers } a d r A \mathrm{~F} \text { and } a d r A \mathrm{R} \text { and inserted } \\
\text { into pGEMT-Easy }\end{array}$ & This study \\
\hline pTadrA::Cm & 5.8-kb Fragment containing adrA::Cm inserted into pGEMT-Easy & This study \\
\hline pTadrA & $\begin{array}{l}\text { 245-bp Fragment containing the } a d r A \text { promoter amplified with primers } a d r A F^{\prime} \mathrm{PP} \text { and } \\
a d r A \mathrm{R} P \mathrm{PP} \text { and inserted into pGEMT-Easy }\end{array}$ & This study \\
\hline $\mathrm{pT} b c s A$ & $\begin{array}{l}\text { 192-bp Fragment containing the } b c s A \text { promoter amplified with primers } b c s A \mathrm{~F}^{\prime} \mathrm{PP} \text { and } \\
b c s A \mathrm{R} P \mathrm{P} \text { and inserted into pGEMT-Easy }\end{array}$ & This study \\
\hline pTagfD & $\begin{array}{l}\text { 754-bp Fragment containing the } a g f D \text { promoter amplified with primers } a g f D F^{\prime} \text { PP and } \\
\text { agfDR PP and inserted into pGEMT-Easy }\end{array}$ & This study \\
\hline $\mathrm{pB} a d r A$ & 245-bp Fragment containing the $a d r A$ promoter, inserted into pBLuescript SK(+/-) & This study \\
\hline $\mathrm{pB} b c s A$ & 192-bp Fragment containing the $b c s A$ promoter, inserted into pBLuescript SK(+/-) & This study \\
\hline $\mathrm{pB} a g f D$ & 754-bp Fragment containing the $a g f D$ promoter, inserted into pBLuescript $\mathrm{SK}(+/-)$ & This study \\
\hline pProbe-GT' & $\mathrm{pVS} 1 / \mathrm{p} 15 \mathrm{a}$ replicon, $g f p$ reporter & Miller et al. 2000 \\
\hline pProbe-GT'::adrA & 245-bp Fragment containing the $a d r A$ promoter, inserted into pPROBE-GT' & This study \\
\hline pProbe-GT'::bcsA & 192-bp Fragment containing the $b c s A$ promoter, inserted into pPROBE-GT' & This study \\
\hline pProbe-GT'::agfD & 754-bp fragment containing the $a g f D$ promoter, inserted into pPROBE-GT' & This study \\
\hline pKD3 & $\mathrm{Cm}^{\mathrm{r}}$, template plasmid carrying cat cassette & Datsenko and Wanner 2000 \\
\hline pGEMT-Easy & $\mathrm{Ap}^{\mathrm{r}}$, lac $Z^{\prime}$ cloning vector & Promega Corp. \\
\hline pBluescript SK & $\mathrm{Cm}^{\mathrm{r}}$, lac $Z^{\prime}$, cloning vector & Stratagene \\
\hline pBBR1 & Cloning vector & Antoine and Locht 1992 \\
\hline
\end{tabular}

${ }^{\mathrm{a}} \mathrm{Cm}^{\mathrm{r}}$ and $\mathrm{Ap}^{\mathrm{r}}=$ chloramphenicol and ampicillin resistant, respectively. 


\section{MATERIALS AND METHODS}

Bacterial strains, media, and culture conditions.

The strains used in this study are listed in Table 3. Bacteria were grown in, or plated on, LB medium (Difco, Becton Dickinson, Franklin Lakes, NJ, U.S.A.). When necessary, antibiotics were used at the following concentrations: ampicillin at $100 \mu \mathrm{g} / \mathrm{ml}$, gentamicin at $25 \mu \mathrm{g} / \mathrm{ml}$, chloramphenicol at 50 $\mu \mathrm{g} / \mathrm{ml}$, and kanamycin at $50 \mu \mathrm{g} / \mathrm{ml}$. Salmonella-Shigella agar (Difco), a Salmonella semiselective indicator medium, was used to determine $S$. enterica populations. Bacterial cultures for biofilm and pellicle assays were suspended in LBNS at approximately $10^{5} \mathrm{CFU} / \mathrm{ml}$ and incubated at $28^{\circ} \mathrm{C}$ in stationary 20-mm test tubes for at least 3 days.

\section{Construction of the adrA mutant.}

To construct plasmids for allelic-exchange mutagenesis, a 4,807-bp fragment containing $a d r A$ was PCR amplified with the primer sets listed in Table 4. The PCR product was cloned into pGEMT-Easy (Promega Corp., Madison, WI, U.S.A.). A chloramphenicol antibiotic resistance gene cassette then was inserted into the NarI site in $a d r A$. This plasmid was electrotransformed into the wild type for allelic-exchange following the methods described (Ried and Collmer 1987). The adrA mutation was confirmed by PCR and Southern hybridization analysis. Transformation, restriction endonuclease digestion, and other DNA techniques were performed essentially as described by Sambrook and Russell (Sambrook et al. 1989).

\section{Alfalfa sprout attachment and colonization assays.}

Alfalfa sprout colonization assays were performed as described previously (Barak et al. 2005). All experiments were performed at least three times. Alfalfa seeds were surface sanitized and sprouted in petri plates, and irrigation water was exchanged daily. Overnight bacterial cultures grown on LB agar were suspended in sterile water at a concentration of approximately $10^{4} \mathrm{CFU} / \mathrm{ml}$ for attachment and colonization assays and at approximately $10^{6} \mathrm{CFU} / \mathrm{ml}$ for microscopy studies. For colonization and microscopy assays, seeds were incubated in the bacterial suspension for $1 \mathrm{~h}$, and then the suspension was replaced with sterile water and the seeds were sprouted for up to 3 days at room temperature. Individual seed or sprouts were rinsed in $1 \mathrm{ml}$ of sterile water for $30 \mathrm{~s}$ and then were homogenized. The homogenate was dilution plated and the cultures were incubated overnight at $37^{\circ} \mathrm{C}$; then, the colonies were enumerated. For attachment assays, seeds were germinated in sterile water for 3 days with daily water changes. After 3 days, sprouts were transferred to $50-\mathrm{ml}$ conical tubes $(12$ sprouts per tube) and incubated in bacterial suspensions for $4 \mathrm{~h}$ at $25^{\circ} \mathrm{C}$ with and constant shaking at $40 \mathrm{rpm}$. Individual sprouts were rinsed in $1 \mathrm{ml}$ of sterile water for $30 \mathrm{~s}$ and then homogenized. The homogenate was dilution plated and the cultures were incubated overnight at $37^{\circ} \mathrm{C}$; then, the colonies were enumerated.

\section{Statistics.}

To determine whether the average populations of bacteria differed, the population data were log transformed and unpaired $t$ tests with Welch correction were performed using GraphPad Instat (version 3.06; GraphPad Software, Inc., San Diego, CA, U.S.A.).

\section{qRT-PCR.}

Alfalfa seeds were surface sanitized and inoculated as described above. The qRT-PCR studies were carried out as described previously (Barak et al. 2005). RNA was extracted from planktonic cells in the sprout irrigation water and bacterial cells attached to plant tissue alfalfa sprouts with the RNeasy kit (Qiagen, Hilden, Germany) following the manufacturer's instructions. DNA was removed using Ambion DNA-free (Ambion, Austin, TX, U.S.A.). The RNA was stored at $-80^{\circ} \mathrm{C}$ until analyzed.

qRT-PCR primers (Operon, Qiagen) are listed in Table 4 along with their target gene. qRT-PCRs were prepared as follows (final volumes per sample): $25 \mu$ of $2 \times$ SYBR green qRT-PCR master mix (Stratagene, La Jolla, CA, U.S.A.), $2 \mu \mathrm{l}$ of each primer $(0.4 \mathrm{mM}), 0.5 \mu \mathrm{l}$ of fluorescein $(10 \mathrm{pM}), 0.1 \mu \mathrm{l}$ of StrataScript RT/Rnase block enzyme mixture (Stratagene), $1 \mu \mathrm{l}$ of RNA template (approximately $200 \mathrm{ng}$ of total RNA), and $19.4 \mu \mathrm{l}$ of Rnase-free water. A four-step RT-PCR protocol was used for the iCycler iQ (BioRad, Hercules, CA, U.S.A.): i) reverse transcriptase $\left(30 \mathrm{~min}\right.$ at $\left.45^{\circ} \mathrm{C}\right)$; ii) denaturation $(10$ $\mathrm{m}$ at $\left.95^{\circ} \mathrm{C}\right)$; iii) amplification and extension program repeated 40 times $\left(30 \mathrm{~s}\right.$ at $95^{\circ} \mathrm{C}, 45 \mathrm{~s}$ at appropriate annealing temperature, and $30 \mathrm{~s}$ at $72^{\circ} \mathrm{C}$, with a single fluorescence measurement); and iv) melting curve program of $1 \mathrm{~m}$ at $95^{\circ} \mathrm{C}, 1 \mathrm{~m}$ at $55^{\circ} \mathrm{C}, 55$ to $80^{\circ} \mathrm{C}$ with a heating rate of $5^{\circ} \mathrm{C}$ per $10 \mathrm{~s}$, and a continuous fluorescence measurement. The identities of the PCR amplicons from the initial assays were confirmed by sequence analysis.

$r p l U$ was used as an internal control for the alfalfa sprout assays and ompA was used for the LBNS static culture assays, because each was constitutively expressed under the conditions examined. To calculate the fold change in gene expression in comparison with the internal control gene, either rplU or ompA, we used the $1.4^{\text {(gene of interest } \mathrm{Ct} \text { - internal control } \mathrm{Ct})}$ method (Pfaffl 2001) and the cycle threshold (Ct) value was determined for each gene at 1,000 relative fluorescent units. For this calculation to be valid, the amplification efficiencies of the target and reference must be approximately equal. They were calculated as $70 \%$ efficiency, thus resulting in the use of 1.4 instead of 2.0 for gene expression fold change.

\section{Microscopy.}

To construct plasmids for reporter assays, putative promoter regions of the $a g f D, b c s A$, and $a d r A$ genes were PCR amplified from $S$. enterica Newport and cloned into pGEMT (Promega Corp.). All constructs were confirmed and promoter orientation determined with PCR and sequencing. Putative promoter

Table 4. PCR primer sets used in this study ${ }^{a}$

\begin{tabular}{|c|c|}
\hline Primers for & Sequences $5^{\prime}-\mathbf{3}^{\prime}$ \\
\hline \multicolumn{2}{|c|}{$a d r A:: \mathrm{Cm}$ mutant construction } \\
\hline $\operatorname{adrAF}$ & TTCAACGATATCAGCGGAGCTCATCTGCACG \\
\hline $\operatorname{adrAR}$ & AAGAAAAGCGAACGCCTGAGCTCTAAAGCC \\
\hline \multicolumn{2}{|c|}{ bcsA compliment construction } \\
\hline bcs $A$-fwd-1145 & GACTAGTGGCGACAAAACGTCCGCCGG \\
\hline bcsA-rev-3797 & CGAGCTCCATTGTTGAGCCTGAGCCATAA \\
\hline \multicolumn{2}{|l|}{ RT-PCR studies } \\
\hline $\operatorname{ardA}$ fwd $28 \mathrm{Q}$ & CTGCCGCCGCGCTGTTTATGATGATGAG \\
\hline$a d r A$ rev $30 \mathrm{Q}$ & TTACCCAGGCGAACAGAAGCGGATACAACA \\
\hline$b c s A 2 \mathrm{RT} F$ & GTCCCACATATCGTTACCGTCCTG \\
\hline$b c s A 2 \mathrm{RT} \mathrm{R}$ & CGCCGCATCATTTCTTCTCCC \\
\hline ompA F & CCTGGTACGCTGGTGCTAAAC \\
\hline ompA R & CGCCATTGATGTTGTCGCCTTTG \\
\hline$r p l U \mathrm{~F}$ RT & CTGTTGAGTTCGCTGAAGTGC \\
\hline$r p l U \mathrm{R}$ RT & CAGCATTTGTGATAGCATTCGTCG \\
\hline \multicolumn{2}{|l|}{ pProbe constructs } \\
\hline $\operatorname{adr} A \mathrm{~F}^{\prime} \mathrm{PP}$ & TCACTCAACCGCTTTTCGGTAAA \\
\hline$a d r A \mathrm{R} P \mathrm{P}$ & ACAGCAGAAAATGCGTGAATG \\
\hline bcs $A \mathrm{~F}^{\prime} \mathrm{PP}$ & TCATCATTGTTATACATTCTGGTTGCTCC \\
\hline bcsAR PP & AAACGCATCAGATTTAGCATGTG \\
\hline $\operatorname{agfDF^{\prime }} \mathrm{PP}$ & TCATGACCATGACTACTATGGACTTCA \\
\hline agfDR PP & AAATTATCTATTACCTTGTTAGCGAC \\
\hline
\end{tabular}

${ }^{\mathrm{a}} \mathrm{PCR}=$ polymerase chain reaction; $\mathrm{RT}=$ reverse-transcriptase. 
regions were excised with SpeI/SacII and cloned into pBluescript (Stratagene). Promoters in pBluescript plasmids were digested with SpeI/SacII and cloned into pProbe GT' (Miller et al. 2000).

Alfalfa seeds were inoculated with approximately $10^{6}$ $\mathrm{CFU} / \mathrm{ml}$ of water as described above and examined at $48 \mathrm{hpi}$ with a Leica TCS NT confocal microscope equipped with AR, $\mathrm{Kr}$, and $\mathrm{He} / \mathrm{Ne}$ lasers (Leica Microsystems, Heidelberg, Germany). Images were obtained and modified by using Leica TCS NT software (version 2.0; Leica Microsystems). The sprouts were washed once with sterile water, blocked in phosphate-buffered saline (PBS) with $1 \%(\mathrm{wt} / \mathrm{vol})$ bovine serum albumen (BSA), and incubated with purified anti-Salmonella CSA-1 antibody (Kirkegaard \& Perry Laboratories, Inc, Gaithersburg, MD, U.S.A.) diluted 1:200 in PBS with 1\% BSA. Sprouts were washed three times with PBS and incubated with the secondary antibody conjugated to Alexa Fluor 568 (Molecular Probes Inc., Eugene, OR, U.S.A.). In order to visualize sprout cells, the inoculated sprouts were stained with $5 \mathrm{mM}$ Syto 59 (Molecular Probes Inc.) in PBS. An Ar laser (excitation wavelength, $488 \mathrm{~nm}$ ) was used to excite the GFP, a $\mathrm{Kr}$ laser (excitation wavelength, $568 \mathrm{~nm}$ ) was used to excite the Alexa Fluor 568-conjugated antibody, and an He/Ne laser (excitation wavelength, $633 \mathrm{~nm}$ ) was used to excite the Syto 59. GFP fluorescence was detected with a BP525/50 filter set and assigned the color green. Alexa Fluor 568 fluorescence was detected with a BP600/30 filter set and assigned the color red. SYTO 59 was detected with a LP645 filter set and assigned the color blue. Three-color images were obtained by overlaying images from the green, red, and blue channels. All microscopy experiments were repeated at least two times, and multiple samples were examined each time.

\section{ACKNOWLEDGMENTS}

This work was supported by the United States Department of Agriculture (Agricultural Research Service CRIS project number 5325-42000044-00D). We would like to thank A. Bates for help with microscopy studies, B. He Shen for construction of the $b \operatorname{cs} A$ compliment plasmid, W. Kay for providing the $S$. enterica Enteritidis strains used in the study, and L. Gorski for helpful critique of the manuscript.

\section{LITERATURE CITED}

Antoine, R., and Locht, C. 1992. Isolation and molecular characterization of a novel broad-host-range plasmid from Bordetella bronchiseptica with sequence similarities to plasmids from gram-positive organisms. Mol. Microbiol. 6:1785-1799.

Ausmees, N., Jonsson, H., Hoglund, S., Ljunggren, H., and Lindberg, M. 1999. Structural and putative regulatory genes involved in cellulose synthesis in Rhizobium leguminosarum bv. trifolii. Microbiology 145:1253-1262.

Barak, J. D., Gorski, L., Naraghi-Arani, P., and Charkowski, A. O. 2005. Salmonella enterica virulence genes are required for bacterial attachment to plant tissue. Appl. Environ. Microbiol. 71:5685-5691.

Beuchat, L. R. 2002. Ecological factors influencing survival and growth of human pathogens on raw fruits and vegetables. Microbes Infect. 4:413423.

Campbell, J. V., Mohle-Boetani, J., Reporter, R., Abbott, S., Farrar, J., Brandl, M., Mandrell, R., and Werner, S. B. 2001. An outbreak of Salmonella serotype Thompson associated with fresh cilantro. J. Infect. Dis. 183:984-987.

Charkowski, A. O., Barak, J. D., Sarreal, C. Z., and Mandrell, R. E. 2002. Differences in growth of Salmonella enterica and Escherichia coli O157:H7 on alfalfa sprouts. Appl. Environ. Microbiol. 68:3114-3120.

Collinson, S. K., Emody, L., Muller, K. H., Trust, T. J., and Kay, W. W. 1991. Purification and characterization of thin, aggregative fimbriae from Salmonella enteritidis. J. Bacteriol. 173:4773-4781.

Datsenko, K. A., and Wanner, B. L. 2000. One-step inactivation of chromosomal genes in Escherichia coli $\mathrm{K}-12$ using PCR products. Proc. Natl. Acad. Sci. U.S.A. 97:6640-6645.

Garcia, B., Latasa, C., Solano, C., Garcia-del Portillo, F., Gamazo, C., and
Lasa, I. 2004. Role of the GGDEF protein family in Salmonella cellulose biosynthesis and biofilm formation. Mol. Microbiol. 54:264-277.

Gibson, D. L., White, A. P., Snyder, S. D., Martin, S., Heiss, C., Azadi, P., Surette, M., and Kay, W. W. 2006. Salmonella produces an O-antigen capsule regulated by AgfD and important for environmental persistence. J. Bacteriol. 188:7722-7730.

Guo, X., Chen, J., Brackett, R. E., and Beuchat, L. R. 2001. Survival of Salmonellae on and in tomato plants from the time of inoculation at flowering and early stages of fruit development through fruit ripening. Appl. Environ. Microbiol. 67: 4760-4764.

Hammar, M., Bian, Z., and Normark, S. 1996. Nucleator-dependent intercellular assembly of adhesive curli organelles in Escherichia coli. Proc. Natl. Acad. Sci. U.S.A. 93:6562-6566.

Hopkins, R. S., Jajosky, R. A., Hall, P. A., Adams, D. A., Connor, F. J., Sharp, P., Anderson, W. J., Fagan, R. F., Aponte, J. J., Nitschke, D. A., Worsham, C. A., Adekoya, N., and Chang, M. H. 2005. Summary of notifiable diseases-United States, 2003. MMWR Morb. Mortal. Wkly. Rep. 52:1-85.

Inami, G. B., and Moler, S. E. 1999. Detection and isolation of Salmonella from naturally contaminated alfalfa seeds following an outbreak investigation. J. Food Prot. 62:662-664.

Jeter, C., and Matthysse, A. G. 2005. Characterization of the binding of diarrheagenic strains of $E$. coli to plant surfaces and the role of curli in the interaction of the bacteria with alfalfa sprouts. Mol. Plant-Microbe Interact. 18:1235-1242.

Ledeboer, N. A., and Jones, B. D. 2005. Exopolysaccharide sugars contribute to biofilm formation by Salmonella enterica serovar typhimurium on HEp-2 cells and chicken intestinal epithelium. J. Bacteriol. 187:3214-3226.

Ledeboer, N. A., Frye, J. G., McClelland, M., and Jones, B. D. 2006. Salmonella enterica serovar Typhimurium requires the Lpf, Pef, and Tafi fimbriae for biofilm formation on HEp-2 tissue culture cells and chicken intestinal epithelium. Infect. Immun. 74:3156-3169.

Mahon, B. E., Ponka, A., Hall, W. N., Komatsu, K., Dietrich, S. E., Siitonen, A., Cage, G., Hayes, P. S., Lambert-Fair, M. A., Bean, N. H., Griffin, P. M., and Slutsker, L. 1997. An international outbreak of Salmonella infections caused by alfalfa sprouts grown from contaminated seeds. J. Infect. Dis. 175:876-882

Matthysse, A. G., and McMahan, S. 1998. Root colonization by Agrobacterium tumefaciens is reduced in cel, attB, attD, and attR mutants. Appl. Environ. Microbiol. 64:2341-2345.

Miller, W. G., Leveau, J. H., and Lindow, S. E. 2000. Improved gfp and inaZ broad-host-range promoter-probe vectors. Mol. Plant-Microbe Interact. 13:1243-1250.

Mohle-Boetani, J. C., Reporter, R., Werner, S. B., Abbott, S., Farrar, J., Waterman, S. H., and Vugia, D. J. 1999. An outbreak of Salmonella serogroup Saphra due to cantaloupes from Mexico. J. Infect. Dis. 180:1361-1364.

O’Mahony, M., Cowden, J., Smyth, B., Lynch, D., Hall, M., Rowe, B., Teare, E. L., Tettmar, R. E., Rampling, A. M., Coles, M., Gilbert, R. J., Kingcott, E., and Bartlett, C. L. R. 1990. An outbreak of Salmonella saint-paul infection associated with beansprouts. Epidemiol. Infect. 104:229-235

Pfaffl, M. W. 2001. A new mathematical model for relative quantification in real-time RT-PCR. Nucleic Acids Res. 29:e45.

Prigent-Combaret, C., Prensier, G., Thi, T. T. L., Vidal, O., Lejeune, P., and Dorel, C. 2000. Development pathway for biofilm formation in curli-producing Escherichia coli strains: role of flagella, curli, and colanic acid. Environ. Microbiol. 2:450-464.

Ried, J. L., and Collmer, A. 1987. An $n p t \mathrm{I}-s a c \mathrm{~B}-s a c \mathrm{R}$ cartridge for constructing directed, unmarked mutations in gram-negative bacteria by marker exchange-eviction mutagenesis. Gene 57:239-246.

Romling, U. 2005. Characterization of the rdar morphotype, a multicellular behaviour in Enterobacteriaceae. Cell Mol. Life Sci. 62:1234-1246.

Romling, U., Sierralta, W. D., Eriksson, K., and Normark, S. 1998. Multicellular and aggregative behaviour of Salmonella typhimurium strains is controlled by mutations in the agfD promoter. Mol. Microbiol. 28:249264.

Romling, U., Rohde, M., Olsen, A., Normark, S., and Reinkoster, J. 2000. $A g f D$, the checkpoint of multicellular and aggregative behaviour in Salmonella typhimurium regulates at least two independent pathways. Mol. Microbiol. 36:10-23.

Romling, U., Bokranz, W., Rabsch, W., Zogaj, X., Nimtz, M., and Tschape, H. 2003. Occurrence and regulation of the multicellular morphotype in Salmonella serovars important in human disease. Int. J. Med. Microbiol. 293:273-285.

Romling, U., Gomelsky, M., and Galperin, M. Y. 2005. C-di-GMP: the dawning of a novel bacterial signalling system. Mol. Microbiol. 57:629639.

Sambrook, J., Fritsch, E. F., and Maniatis, T. 1989. Molecular Cloning: A 
Laboratory Manual. Cold Spring Harbor Laboratory Press, Plainview, NY, U.S.A

Scher, K., Romling, U., and Yaron, S. 2005. Effect of heat, acidification, and chlorination on Salmonella enterica serovar typhimurium cells in a biofilm formed at the air-liquid interface. Appl. Environ. Microbiol. 71:1163-1168.

Simm, R., Morr, M., Kader, A., Nimtz, M., and Romling, U. 2004. GGDEF and EAL domains inversely regulate cyclic di-GMP levels and transition from sessility to motility. Mol. Microbiol. 53:1123-1134.

Solano, C., Garcia, B., Valle, J., Berasain, C., Ghigo, J. M., Gamazo, C., and Lasa, I. 2002. Genetic analysis of Salmonella enteritidis biofilm formation: critical role of cellulose. Mol. Microbiol. 43:793-808.

White, A. P., Collinson, S. K., Banser, P. A., Gibson, D. L., Paetzel, M., Strynadka, N. C., and Kay, W. W. 2001. Structure and characterization of AgfB from Salmonella enteritidis thin aggregative fimbriae. J. Mol Biol. 311:735-749.
White, A. P., Gibson, D. L., Collinson, S. K., Banser, P. A., and Kay, W. W. 2003. Extracellular polysaccharides associated with thin aggregative fimbriae of Salmonella enterica serovar enteritidis. J. Bacteriol. 185:5398-5407.

White, A. P., Gibson, D. L., Kim, W., Kay, W. W., and Surette, M. G. 2006. Thin aggregative fimbriae and cellulose enhance long-term survival and persistence of Salmonella. J. Bacteriol. 188:3219-3227.

Winthrop, K. L., Palumbo, M. S., Farrar, J. A., Mohle-Boetani, J. C., Abbott, S., Beatty, M. E., Inami, G., and Werner, S. B. 2003. Alfalfa sprouts and Salmonella Kottbus infection: a multistate outbreak following inadequate seed disinfection with heat and chlorine. J. Food Prot. 66:13-17.

Zogaj, X., Nimtz, M., Rohde, M., Bokranz, W., and Romling, U. 2001 The multicellular morphotypes of Salmonella typhimurium and Escherichia coli produce cellulose as the second component of the extracellular matrix. Mol. Microbiol. 39:1452-1463. 